\title{
Fammab
}

FERMILAB-Conf-03/213 July 2003

\section{Collider and Detector Protection at Beam Accidents*}

\author{
I.L. Rakhno, N.V. Mokhov, A.I. Drozhdin \\ FNAL, Batavia, IL 60510, USA
}

July 18, 2003

\begin{abstract}
Dealing with beam loss due to abort kicker prefire is considered for hadron colliders. The prefires occured at Tevatron (Fermilab) during Run I and Run II are analyzed and a protection system implemented is described. The effect of accidental beam loss in the Large Hadron Collider (LHC) at CERN on machine and detector components is studied via realistic Monte Carlo calculations. The simulations show that beam loss at an unsynchronized beam abort would result in severe heating of conventional and superconducting magnets and possible damage to the collider detector elements. A proposed set of collimators would reduce energy deposition effects to acceptable levels. Special attention is paid to reducing peak temperature rise within the septum magnet and minimizing quench region length downstream of the LHC beam abort straight section.
\end{abstract}

*Presented paper at the ICFA Workshop on Beam Halo Dynamics, Diagnostics, and Collimation (HALO'03), Montauk, Long Island, NY, May 19-23, 2003 


\title{
Collider and Detector Protection at Beam Accidents
}

\author{
I. L. Rakhno, N. V. Mokhov, A. I. Drozhdin \\ Fermilab, P.O. Box 500, Batavia, IL 60510, USA
}

\begin{abstract}
Dealing with beam loss due to abort kicker prefire is considered for hadron colliders. The prefires occured at Tevatron (Fermilab) during Run I and Run II are analyzed and a protection system implemented is described. The effect of accidental beam loss in the Large Hadron Collider (LHC) at CERN on machine and detector components is studied via realistic Monte Carlo calculations. The simulations show that beam loss at an unsynchronized beam abort would result in severe heating of conventional and superconducting magnets and possible damage to the collider detector elements. A proposed set of collimators would reduce energy deposition effects to acceptable levels. Special attention is paid to reducing peak temperature rise within the septum magnet and minimizing quench region length downstream of the LHC beam abort straight section.
\end{abstract}

\section{INTRODUCTION}

An accidental beam loss caused by an unsynchronized abort launched at abort system malfunction, can cause severe damage to a collider equipment. Such a malfunction can be initiated, e.g., by a spontaneous high voltage discharge in a kicker generator module or high energy cosmic particle crossing a sensitive element of the abort system trigger. Statistical data accumulated at Tevatron for Run I and Run II indicate that such prefires happened, on the average, a few times a year.

In the LHC a single prefired kicker module induces coherent beam oscillations with an amplitude up to $21 \sigma$ of the beam at collisions. Simulations show that if this happens at the top energy, starting from $70-80 \%$ of the kicker strength, the misbehaved beam ends up in the IP5 inner triplet causing destruction of its components and damage to the CMS detector near-beam elements [1]. To avoid this, the other kicker modules are fired immediately after the prefired one (thus producing a full, unsynchronized, abort), but this does not prevent beam loss completely. A set of stationary collimators for the IP5 has been proposed in [1] to protect its inner triplet against irreversible consequences of a fast beam loss. Alternatively, a movable collimator TCDQ, in IP6 as close to the cause as possible, has been proposed in [2] to protect the entire LHC machine. There were two major unresolved energy deposition problems associated with the effect of an un-

\footnotetext{
1 This work was supported by the Universities Research Association, Inc., under contract DE-AC02-76CH03000 with the U.S. Department of Energy.

2 rakhno@fnal.gov
}

synchronized beam abort in the LHC IP6 [3]: (i) a peak temperature rise at the upstream end of the septum magnet MSD exceeds the limit of $100^{\circ} \mathrm{C}$; (ii) a peak energy deposition in superconducting (SC) coils downstream of the Q5 quadrupole remains above the quench limit in more than $50 \%$ of the magnet string resulting in an unacceptably severe quench. The solutions to these problems has been recently proposed based on the updated LHC beam optics (version 6.4) and detailed MARS [4] calculations.

\section{TEVATRON}

For Tevatron Run I (from December 1993 till February 1996) there were 10 abort kicker prefires (AKP) and "fails-to-fire", while for the initial stage of Run II (from March 2001 till November 2002) there were 7 documented AKP. The average rate of one AKP per about three months is quite high taking into account recovery of the machine after severe quench of the SC magnets and possible damage to sensitive electronics. For example, three recent AKP in Tevatron caused quenches of several SC dipoles in the A-sector and $\mathrm{B} \emptyset$ region and 2Gy instantaneous dose in the CDF central detector that gave rise to damage of silicon ladders.

To protect the CDF detector and Tevatron components in an event of AKP, an existing collimator at the A11 straight section will be used and a new collimator is to be installed at the A48 location during the summer 2003 shutdown $[5,6]$. The A11 collimator will protect the Tevatron dipoles. Detailed calculations with the MARS [4] and STRUCT [7] codes have shown that a 0.5-m long steel collimator at A48 will intercept one of 
36 proton bunches when such an incident occurs, providing reliable protection of the CDF main detector at an AKP. It will also mitigate the backgrounds induced by elastic beam-gas interactions upstream of BØ. Although the Roman Pot detectors downstream of the A48 collimator will see an increased background, the amount of radiation they will receive either resulting from beam halo interactions in the collimator or during an AKP will not damage their sensitive parts.

A bunch lost at A48 during an AKP represents more than $2 \times 10^{11}$ protons "instantaneously" interacting with the collimator material. Although the A11 and A48 collimators protect the downstream SC dipoles against a damage in such an event, secondaries generated in them create a significant radiation load on the dipoles which will likely result in a quench of the first one. Optimization of the A48 collimator based on detailed MARS simulations [6] allows to reduce the peak energy deposition density in the SC coil from 17 to $3.5 \mathrm{~mJ} / \mathrm{g}$ (see Figs. 1 and 2). Due to the space constraints at this location, this is the maximum achievable reduction. The radiation load is reduced, but the first dipole is still subject to quench because the above values exceed the quench limit of $0.5 \mathrm{~mJ} / \mathrm{g}$ per pulse at fast beam loss [8].

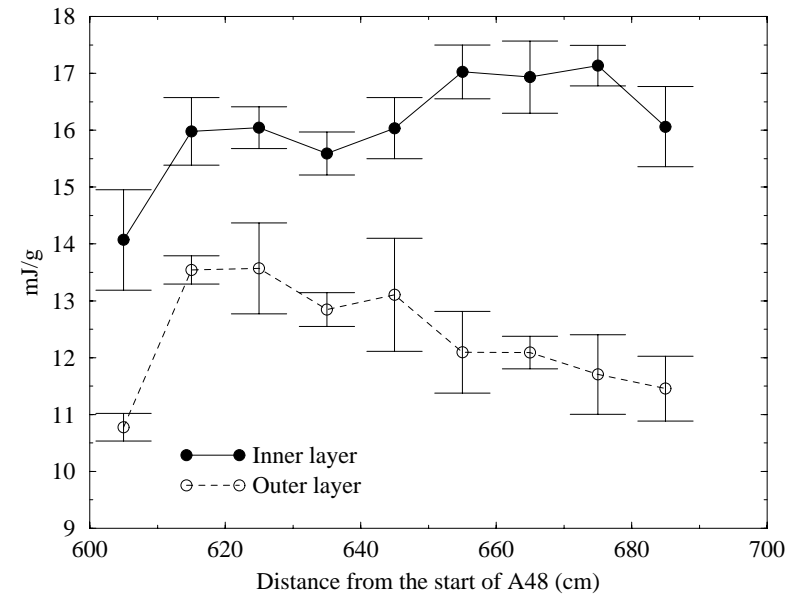

FIGURE 1. Energy deposition in the inner and outer SC coils of the Tevatron A48 first dipole for the baseline 0.5-m stainless steel collimator.

\section{LHC}

\section{IP6 Model and Beam Parameters}

A central part of the current LHC IP6 calculation model, optimized over years in thorough MARS calculations, is shown in Fig. 3. A stationary 5-m long rectangular graphite collimator TCDS is placed at $0.1335 \mathrm{mrad}$ with respect to the circulating beam axis. Its width increases gradually from 24.5 to $25.2 \mathrm{~mm}$ when going from

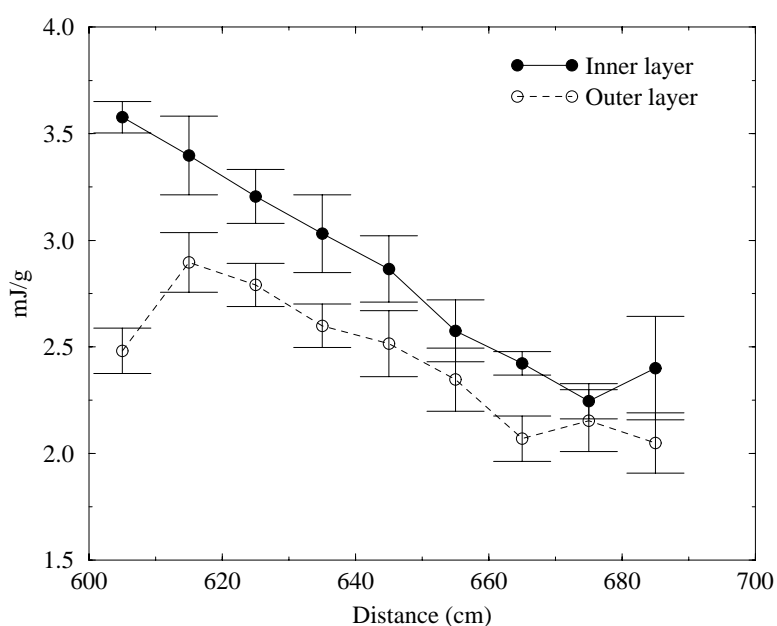

FIGURE 2. Energy deposition in the horizontal plane of the inner and outer SC coils along the Tevatron A48 first dipole for the final collimator arrangement: a composite A48 collimator (0.3-m tungsten followed by $0.37-\mathrm{m}$ stainless steel) with a $0.34-$ $\mathrm{m}$ tungsten mask with a round $2.5-\mathrm{cm}$ radius aperture placed immediately upstream of the first dipole.

a non-IP facet to IP one. The facets are placed at 14 and $14.9 \mathrm{~mm}$ from the circulating beam axis, respectively. A composite $9.5-\mathrm{m}$ long graphite $(8 \mathrm{~m})$ and aluminum $(1.5 \mathrm{~m})$ collimator TCDQ is placed at a radial position of $9.1 \mathrm{~mm}$, corresponding to $8 \sigma_{x}$ of the circulating beam at collision energy of $7 \mathrm{TeV}$, plus orbit deviations. It is movable, i.e. the jaws are retracted at injection to accommodate a larger beam size.

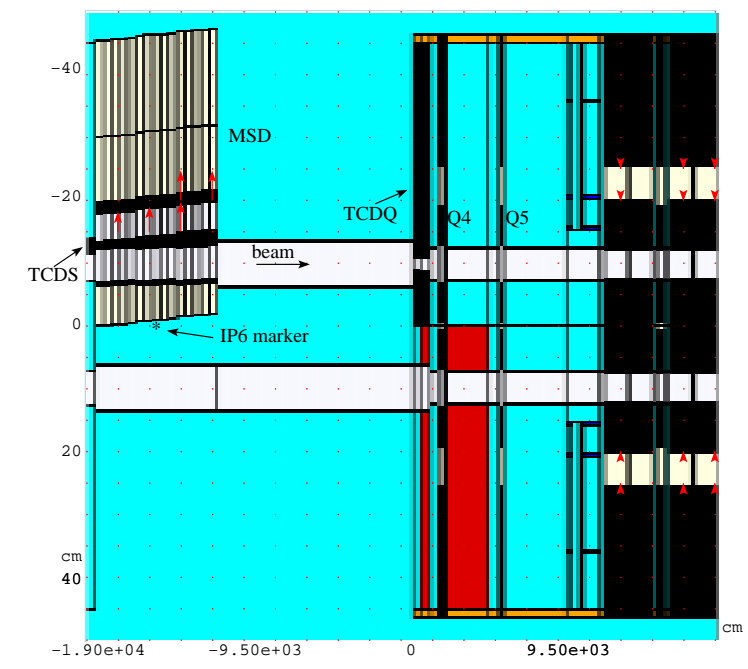

$\vec{Y}^{2}$

FIGURE 3. Central part of the IP6 MARS model.

The beam parameters used are described in Table 1. In our calculations, we assume that bunches are distributed uniformly around the lattice and there is only one $3 \mu \mathrm{s}$ abort gap in the circulating beam. To cover the abort 
kicker rise time of $3 \mu$ s (see Table 1), 280 bunches are considered which correspond to $7 \mu \mathrm{s}$. It enables us to investigate effect of delay time $\tau$, i.e. time elapsed between prefiring the single kicker and firing the other ones. Results presented below were obtained for a kicker strength $B \cdot l=63.05 \mathrm{kG} \cdot \mathrm{m}$, which corresponds to an angle $\alpha=0.27 \mathrm{mrad}$ at $7 \mathrm{TeV}$.

TABLE 1. LHC beam parameters used in the study.

\begin{tabular}{lc}
\hline Proton energy & $7000 \mathrm{GeV}$ \\
Normalized transverse emittance $(\sigma)$ & $3.75 \mathrm{~mm} \cdot \mathrm{mrad}$ \\
Protons per bunch & $1.05 \cdot 10^{11}$ \\
Number of bunches & 2835 \\
Total intensity & $3 \cdot 10^{14}$ \\
Horizontal crossing angle in the IP5 & $150 \mu \mathrm{rad}$ \\
Bunch separation (10 RF buckets) & $24.95 \mathrm{~ns}$ \\
Abort gap (127 missing bunches) & $3.17 \mu \mathrm{s}$ \\
Number of abort kicker modules & 14 \\
Abort kicker rise time & $3 \mu \mathrm{s}$ \\
\hline
\end{tabular}

TABLE 2. Instantaneous peak temperature rise $\Delta \mathrm{T}(\mathrm{C})$ in the collimators and MSDA1 dipole at baseline luminosity.

\begin{tabular}{lrrr}
\hline & \multicolumn{3}{c}{ Delay time $\tau(\mu \mathrm{s})$} \\
\cline { 2 - 4 } \multicolumn{1}{c}{ Module } & 1.2 & 3.0 & \multicolumn{1}{c}{4.0} \\
\hline TCDS $(5 \mathrm{~m})$ & 698 & 680 & 694 \\
MSDA1 $(4.5 \mathrm{~m})$ & 522 & 450 & 504 \\
TCDQ1 $(4 \mathrm{~m})$ & 456 & 810 & 1170 \\
TCDQ2 $(4 \mathrm{~m})$ & 155 & 246 & 348 \\
TCDQ3 $(1.5 \mathrm{~m})$ & 5 & 14 & 34 \\
\hline
\end{tabular}

\section{Temperature Rise in IP6 Components}

The results of previous MARS [2, 3] calculations are summarized in Table 2. A single prefired kicker is not strong enough to deflect the beam significantly for it to hit the TCDS collimator. Deflected bunches hit the collimator when the other fired kickers attain a given strength. That is why almost no dependence of instantaneous peak temperature rise in the TCDS collimator and MSD magnet on delay time is observed in the results. The peak temperatures in the TCDS and TCDQ graphite are well below the shock wave limit of about $2200^{\circ} \mathrm{C}$. The Tevatron beam abort dump operates for more than 20 years with the peak temperature in the graphite core of $1000^{\circ} \mathrm{C}$. At the same time, the peak temperature rise in the septum magnet MSDA1, immediately downstream of the 5-m graphite TCDS collimator, is unacceptably high. Heating a magnet up to about $110^{\circ} \mathrm{C}$ with subsequent cooling down gives rise to irreversible changes in magnetic properties and field homogeneity [9]. The problem can be solved by increasing the graphite length by $1 \mathrm{~m}$ and adding at the TCDS downstream end a $0.5-\mathrm{m}$ long iron section of the same profile as the graphite one. The resulting peak temperature distribution along such a composite 6.5-m TCDS collimator and MSDA magnet is shown in Fig. 4. One sees that the maximum instantaneous temperature rise in the MSDA1 at an unsynchronized beam abort is about $12^{\circ} \mathrm{C}$ which is quite acceptable. This value almost does not depend on the delay time.

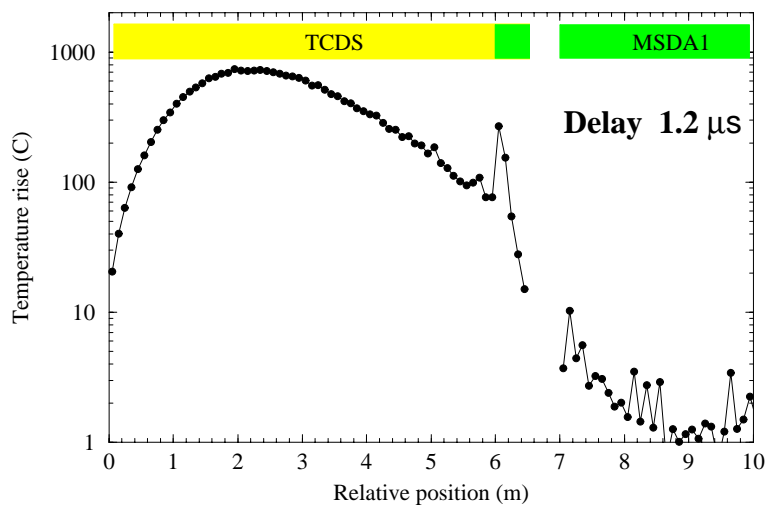

FIGURE 4. Peak temperature rise along the TCDS collimator and MSDA1 magnet at baseline luminosity.

\section{Minimizing Quench Region Length}

The SC magnets in the IP6 and farther in the machine are reliably protected against destruction at an unsynchronized beam abort by the TCDQ collimator proposed in Ref. [2]. There is no easy way to avoid quench of the first SC quadrupoles Q4 and Q5, but what matters is the length of the quenched string afterwards. It is required to limit the number of quenched dipoles to less than $50 \%$ of the string. The peak energy deposition density $\varepsilon_{\max }$ in the SC coils should be compared to the quench limit that can be estimated as $0.5 \mathrm{~mJ} / \mathrm{g}$ per pulse for the LHC magnets at fast beam loss $(\leq 1 \mathrm{~ms})$ [8]. To reduce peak energy deposition $\varepsilon_{\text {max }}$ downstream of Q5, two 2-m steel masks were implemented. The first one, with the aperture of $20 \sigma_{c o l}$, is downstream the Q5 quadrupole and second one, with aperture of $21 \sigma_{c o l}$, is in front of the MBA1. The masks enable one to limit the quench region to one ( $\tau=1.2 \mu \mathrm{sec})$ or two $(\tau=4 \mu \mathrm{sec})$ dipoles with other magnets downstream remaining in the superconducting state (see Fig. 5). It reduces $\varepsilon_{\max }$ in the MBA1 magnet by almost a factor of ten. Unfortunately, the value of $20 \sigma_{\mathrm{col}}$ is less than $10 \sigma_{i n j}$ and that requires for the two masks to be movable ones.

Larger apertures of the masks, if they are fixed, do not give such a significant protection effect. Therefore, other options should be considered in further studies to avoid using movable masks, provide $\varepsilon_{\text {max }}$ in the Q4, Q5, MBA1 and $\mathrm{MBB} 1$ as low as possible and mitigate collimator alignment problem. The possibilities are to split the 9.5- 


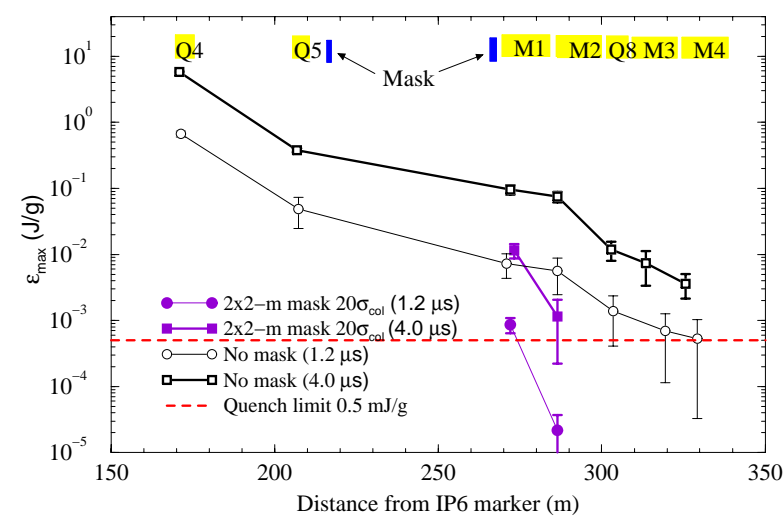

FIGURE 5. Peak energy deposition density $\varepsilon_{\max }$ in SC coils vs. distance from the IP6 marker, where M1 - M4 denote the first four dipoles (MBA1, MBB1 etc).

$\mathrm{m}$ TCDQ collimator into two shorter sections with about $10 \mathrm{~m}$ between them, and to use a V-shaped aperture for a stationary TCDQ with separate passes for injected and $7-\mathrm{TeV}$ beams by means of a constant field dogleg.

The radiation loads in the rest of the machine downstream of the M4 dipole are well below the limits, with undetectable beam loss rate at all critical locations.

\section{Radiation Levels in Tunnel}

An unsynchronized beam abort gives rise to instantaneous irradiation in the tunnel. Distributions of equivalent dose can be useful to estimate dose load to monitoring electronics during such an accident and determine the most dangerous regions. The distributions due to a beam abort have been calculated with MARS in the tunnel in the vicinity of the vessel and near the farthest concrete wall. The extracted beam is supposed to be directed to an external beam dump and, therefore, contribution to dose in the tunnel due to the beam was not considered. According to the calculations, the hottest spots in the tunnel are near the TCDS and TCDQ collimators: for a $1.2-\mu \mathrm{s}$ delay time the instantaneous dose in the vicinity of the vessel equals to 800 and $100 \mathrm{~Sv}$, respectively. The instantaneous dose around other components in the region is substantially lower: 0.1 to $1 \mathrm{~Sv}$.

\section{CONCLUSIONS}

The performed studies revealed that for both Tevatron and LHC the proposed collimators ensure reliable protection against destruction (melting down) of the SC coils and detector components due to abort kicker prefire, and drastically reduce the overall radiation loads. How- ever, in both the cases the first SC magnets immediately downstream of such collimators will quench. The number of such magnets is one at Tevatron and seven at LHC. Possibilities to reduce the number of the magnets subject to quench are under investigation. The studies revealed that, with the LHC collimators TCDS and TCDQ in the appropriate position, the machine and detector components are reliably protected against any damage at an unsynchronized beam abort with the peak temperature rise in the IP6 components being quite acceptable. A reevaluation of the peak energy deposition is required to take into account the recently confirmed shorter delay time of $0.7 \mu$ s instead of the previously assumed $1.2 \mu \mathrm{s}$. Slow (continuous) beam loss on TCDQ is of interest in the case it becomes a secondary collimator.

\section{ACKNOWLEDGMENTS}

Authors thank B. Goddard, M. Gyr, L. Nicolas, R. Schmidt and D. Still for useful discussions.

\section{REFERENCES}

1. Drozhdin, A. I., Mokhov, N. V., and Huhtinen, M., "Impact of the LHC Beam Abort Kicker Prefire on High Luminosity Insertion and CMS Detector Performance," in Particle Accelerator Conference-1999, IEEE Conference Proceedings 99CH36366, IEEE, New York, 1999, pp. 1231-1233.

2. Mokhov, N. V., Drozhdin, A. I., Rakhno, I. L., Gyr, M., and Weisse, E., "Protecting LHC Components Against Radiation Resulting from an Unsynchronized Beam Abort," in Particle Accelerator Conference-2001, IEEE Conference Proceedings 01CH37268, IEEE, Chicago, 2001, pp. 3168-3170.

3. Rakhno, I. L., Drozhdin, A. I., Mokhov, N. V., Goddard, B., Gyr, M., Sans, M., and Weisse, E., Further Studies on Protecting LHC Components against Radiation resulting from an Unsynchronized Beam Abort, Tech. rep., Fermilab-FN-724 (2002).

4. Mokhov, N. V., Status of MARS Code, Tech. rep., Fermilab-Conf-03/053 (2003).

5. Church, M. D., Drozhdin, A. I., Moore, R. S., and Still, D. A., Tevatron Abort Kicker Prefire Simulations, Tech. rep., Fermilab Beams-doc-648 (2003).

6. Nicolas, L. Y., and Mokhov, N. V., Impact of the A48 Collimator on the Tevatron B0 Dipoles, Tech. rep., Fermilab-TM-2214 (2003).

7. Baishev, I. S., Drozhdin, A. I., and Mokhov, N. V., STRUCT Program User's Reference Manual, Tech. rep., SSCL-MAN-0034 (1994).

8. Fermilab Superconducting Accelerator Design Report, Tech. rep., Fermilab (1979).

9. Rijk, G. D., Private Communication, CERN (2002). 